\title{
Role of Endoluminal Catheter Colonization Surveillance Cultures to Reduce Catheter-Related Blood Stream Infections in Hemodialysis Patients
}

Soheir Abd El- Rahman ${ }^{\text {a }}$, Afaf Fathi Khamiss ${ }^{a}$, Saddam H. Abd El- Aziz ${ }^{\text {b }}$, Hala A. Abd El- Karim ${ }^{\text {c }}$

a Department of clinical and chemical pathology, Benha faculty of medicine, Banha University, Egypt. ${ }^{\mathrm{b}}$ Department of internal medicine, Benha faculty of medicine, Banha University, Egypt. ${ }^{\mathrm{c}}$ Department of Nephrology and Urology, Mit ghamr hospital, Ministry of Health, Mansoura, Egypt.

Correspondence to: Hala A. Abd El- Karim, Department of Nephrology and Urology, Mit ghamr hospital, Ministry of Health, Mansoura, Egypt

Email:

hala.mostafa@fmed.bu.edu.eg

Received: 4 January, 2020

Accepted:23 February, 2020

\begin{abstract}
:
Background: Central Venous Catheter (CVC) use as vascular access to the systemic circulation is highly vital for Hemodialysis (HD) patients. Catheter-related bloodstream infection (CRBSI) is the main complication of CVC use. Aim of the work: To assess the use of surveillance cultures (SCs) to prevent CRBSIs in asymptomatic HD patients. Patients and Methods: A prospective cohort study was conducted on eighteen HD patients with CVC, admitted to Dialysis Units of Benha University Hospitals and Meet Ghamr Nephrology and Urology Hospital during the period from October 2016 to March 2017. Endoluminal colonization of the catheter was assessed every 15 days by inoculating $\sim 5-10 \mathrm{~mL}$ of endoluminal blood into aerobic blood culture bottles. Individual patients were triaged based on SC results: group 1 (negative); group 2 (coagulase-negative Staphylococcus [CoNS] with time-topositivity (TTP) $>14$ hours); group 3 (CoNS with TTP $\leq 14$ hours); and group 4 (any microorganism other than CoNS with any TTP or CoNS with Differential Time to Positivity (DTP) more than 120
\end{abstract} minutes). Results: A total of 60 SCs were collected with a mean number per patient of 3.3. Of which 24 SCs (40\%) were negative (group 1), and 36 SCs (60\%) were positive (0 in group 2, 6 in group 3, and 30 in group 4). Under this protocol, the incidence density of CRBSI was 10.8 episodes per 1000 catheter days. Conclusion: SCs based on easily accessible samples proved useful in triaging HD patients at a high risk of infection.

Keywords: Central Venous Catheter, Catheter-related bloodstream infection, Surveillance cultures. List of Abbreviations: CVC, HD, CRBSI, SCs, TTP, CoNS, ALT 



\section{Introduction:}

HD requires long-term and effective vascular access, preferably via arteriovenous fistulas or grafts. However, for many reasons, including old age and comorbidities, the proportion of patients who undergo HD with a central venous catheter is growing, and these patients are at increased risk of infection [1].

Furthermore, infection is a major cause of hospitalization in HD patients. In the United States, the infection was observed in about $30 \%$ of all hospitalizations, was of HD patients. These data show that infection is a serious threat to these cases [2].

Current estimates are that between $15 \%$ and $30 \%$ of all nosocomial bacteremia is catheter-care protocol. However, the high false-positive rates make to discourage it as a CRBSI preventive strategy. Few studies have examined the usefulness of endoluminal surveillance culture (SC) to prevent CRBSI [1]. We thereby conducted a cohort study of HD patients to assess SCs of readily accessible endoluminal samples to decrease the rate of CRBSI and avoid widespread administration of Antibiotic Lock Therapy (ALT) post-dialysis. catheter-related. CRBSIs have significant associated morbidity, increased hospital costs, estimated at 18,000 Euros per episode, and increased length of stay. Attributable mortality ranges between $12 \%$ and $25 \%[3]$. Colonization of the intraluminal surface of the catheter occurs in a high percentage of HD cases.

Endoluminal colonization is a step in the pathogenesis of CRBSIs and precedes many symptoms of peripheral bacteremia and sepsis [1]. The presence of a biofilm in the catheter lumen is one of the factors that complicate the infection treatment [4].

Exit-site surveillance cultures have been investigated in several studies as part of the

\section{Patients and Methods:}

\section{Study population:}

We conducted this prospective cohort study between October 2016 and March 2017 at Microbiology Unit, Clinical Pathology Departments at Benha University Hospitals, with the approval of the ethics committee of the institution. 
The study included 18 patients with central venous catheterization admitted to Dialysis Unit in Benha University Hospitals and Meet Ghamr Nephrology and Urology Hospital. The patients enrolled in this study were $>18$ years of age with end-stage renal disease and underwent HD with a dual lumen central venous catheter and who attended the concerned dialysis units. Patients with a CVC since the start of HD and patients who underwent $\mathrm{CVC}$ insertion during the study period were included in the study. Patients left the study when their CVCs were permanently removed (because of implementation of an arteriovenous fistula or renal transplantation), when they were transferred to another HD center, for prolonged hospitalization (>15 days), or because they died. Demographic and clinical information were collected prospectively on all patients.

\section{Infection control measures:}

Patients were treated with HD sessions 2 or 3 times a week. The dialysis equipment was connected directly to the catheter hub, and the catheter was opened for the shortest time possible to minimize the risk of intraluminal infection. The catheter hub was closed with heparin after each dialysis session. Chlorhexidine was used as an antiseptic agent, and maximum sterile barriers (sterile gloves, masks, hair caps) were used during the connection and disconnection of lines.

\section{Method [1]:}

Colonization of the inner catheter lumen was assessed every 15 days by (SCs) performed on $5-10 \mathrm{~mL}$ of blood and heparin mixture that was withdrawn from the arterial catheter lumen and inoculated into aerobic culture bottles, which were incubated for 7 days in an automated blood culture system (BacT/Alert, bioMèrieux, Durham, NC). This blood and heparin mixture is readily accessible and is normally withdrawn and discarded by the nursing staff just before connecting the patient to the HD machine.

Time-to positivity (TTP), defined as the duration between the start of culture incubation and the start of the alert signal (as documented by the monitoring system), was recorded.

Based on SCs results, triage of individual asymptomatic HD patients was done. When SCs were negative (group 1), if CoNS with TTP >14 hours (group 2), If CoNS was recovered with TTP $<14$ hours (group 3) \& Recovery of microorganisms other than CoNS (S aureus, Enterococcus spp, gramnegative bacteria, Candida spp) with any TTP or CoNS with DTP more than 120 
minutes was considered significant, and a diagnosis of CRBSI was suspected (group 4).

Different treatment strategies were adopted based on the presence or absence of microbial growth, organism identification, and TTP. When SCs were negative (group 1), we Considered these patients at no risk of infection; therefore, no special measures were followed. When CoNS was recovered with TTP >14 hours (group 2), we determined whether it was caused by catheter colonization or culture contamination by performing a fresh SC after 1 week. If the same organism was recovered at the second withdrawn culture, ALT was implemented. If CoNS was recovered with TTP $\leq 14$ hours (group 3), we assumed it was colonizing the catheter, and ALT were also administered. Recovery of microorganisms other than CoNS (S aureus, Enterococcus spp, gram-negative bacteria, Candida spp) or CoNS with DTP more than 120 minutes was considered significant, and a diagnosis of CRBSI was suspected (group 4). In these cases, blood cultures from a peripheral vein and the catheter were setup, and patients were managed according to therapeutic guidelines.

\section{Definitions [5]:}

\section{CRBSI:}

A positive peripheral blood culture in which the microorganism isolated was identical in species and antibiogram to the hub blood culture and the hub blood culture yielded positive results at least 120 min earlier than the peripheral blood cultures.

\section{Non-CRBSI:}

Positive peripheral blood culture and the hub blood culture was either negative or positive and give the same pathogen as peripheral blood culture, but the DTP of the cultures was shorter than $120 \mathrm{~min}$.

We could consider catheter colonization when the hub blood culture was positive but the pathogen isolated was different from the one isolated in peripheral blood cultures or when peripheral blood cultures were negative. For the study analysis, we included these cases in the general non-CRBSI diagnosis.

\section{DTP:}

The differential time to positivity is defined as the difference between the time a blood culture from the catheter becomes positive and the positivity time of peripheral blood culture. 


\section{Statistical analysis:}

The rate of CRBSI was calculated as a density of incidence and was reported per 1,000 catheter days. The collected data were tabulated and analyzed using SPSS version 16 software (SPSS Inc., Chicago,

Illinois, USA). Categorical data were presented as numbers and percentages. McNemer's test and Fisher's exact test (FET) were used to analyze categorical variables. Quantitative data were tested for normality using the Shapiro-Wilks test assuming normality at $\mathrm{P}>0.05$. Quantitative data were expressed as mean \pm standard deviation and range if normally distributed or median and IQR if not. Student "t" test was used to analyze normally distributed variables among 2 independent groups. The accepted level of significance in this work was stated at 0.05 ( $P<0.05$ was considered significant $)$.

\section{Results:}

\section{Study population:}

This study started with 24 patients with CVC admitted to dialysis units in Benha University Hospital and Meet Ghamr Nephrology and Urology Hospital. Six patients left the study: 3 patients $(50 \%)$ were transferred to other centers, in 2 patients $(33.3 \%)$ the catheter was removed in an unexpected time and one patient $(16.7 \%)$ died. So, the study was completed with 18 patients admitted to the dialysis unit during the period from October 2016 to March 2017.

Table (1): Socio-demographic characters of the study population.

\begin{tabular}{llcc}
\hline Variable & & $\begin{array}{c}\text { No. } \\
(\mathbf{N}=\mathbf{1 8})\end{array}$ & $\begin{array}{c}\text { \% } \\
(\mathbf{1 0 0 \%})\end{array}$ \\
\hline Sex & Male & 9 & 50.0 \\
& Female & 9 & 50.0 \\
Age (years) & Mean \pm SD & $54.4 \pm 11$ \\
& Min. - max & \multicolumn{2}{c}{$29-73$} \\
& (Range) & & \\
Site of & Internal & 17 & 94.4 \\
catheter & jugular vein & & \\
& Femoral & 1 \\
& vein & \multicolumn{2}{|c}{5.6} \\
Duration of & Mean \pm SD & $50.6 \pm 13.1$ \\
catheterization & Min. - max & \multicolumn{2}{|c}{$32-71$} \\
(days) & (Range) & \\
\hline
\end{tabular}

\section{Results of Surveillance cultures:}

The selected patients were subjected to SCs regularly every 15 days (from the central line). The incidence of CRBSI in our study was 10.8 per 1000 catheter days. A total of 60 SCs were collected with a mean number per patient of $3.3 \mathrm{SCs}$. Of these $24 \mathrm{SCs}$ (40\%) were negative (group 1), and 36 SCs (60\%) gave positive results. The TTP for blood cultures was identified and the 
positive cultures were classified into different groups as follow: $0(0 \%)$ in group 2 (CoNS with TTP > 14 hours), 6 (10\%) in group 3(CoNS with TTP </= 14 hours), and $30(50 \%)$ in group 4 (microorganisms other than CoNS with any TTP or CoNS with DTP more than 120 minutes). In group 3, three catheters were locked with ALT according to culture and sensitivity results and according to each institute guidelines. All catheters were successfully sterilized, and systemic therapy was not required for these patients. In group 4, all patients were finally diagnosed as CRBSI.

Table (2): Final Diagnosis of patients according to $\mathrm{SC}$ results

\begin{tabular}{|c|c|c|c|c|}
\hline \multicolumn{2}{|c|}{ Variable } & \multirow{2}{*}{$\begin{array}{c}\begin{array}{c}\text { No. of } \\
\text { patients } \\
(\mathbf{N}=\mathbf{1 8})\end{array} \\
10\end{array}$} & \multirow{2}{*}{$\begin{array}{c}\begin{array}{c}\% \\
(100 \%)\end{array} \\
55.6\end{array}$} & \multirow{2}{*}{$\begin{array}{c}\begin{array}{c}\text { Group } \\
\text { classification }\end{array} \\
4\end{array}$} \\
\hline \multirow{4}{*}{ 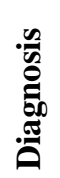 } & CRBSI & & & \\
\hline & Colonization & 3 & 16.7 & 3 \\
\hline & Contamination & 0 & 0 & 2 \\
\hline & Negative & 5 & 27.7 & 1 \\
\hline
\end{tabular}

Ten cases were diagnosed as CRBSI and the causative microorganisms were $\mathrm{S}$ aureus (n $=4), \operatorname{CoNS}(\mathrm{n}=4)$, Acinetobacter $(\mathrm{n}=1)$ and $\mathrm{E}$ coli $(\mathrm{n}=1)$.

3 patients developed colonization of their catheters and the detected microorganisms were $S$ aureus $(n=2)$ and CoNS $(n=1)$.
Table (3): Characteristics of HD patients by CRBSI status during the study period.

\begin{tabular}{|c|c|c|c|c|}
\hline Variable & & $\begin{array}{c}\text { Patients } \\
\text { with } \\
\text { CRBSI } \\
(n=10)\end{array}$ & $\begin{array}{c}\text { Patients } \\
\text { with } \\
\text { Non } \\
\text { CRBSI } \\
(\mathbf{n}=8)\end{array}$ & $\begin{array}{l}P \\
\text { Value* }\end{array}$ \\
\hline Age(years) & $\begin{array}{l}\text { Mean } \pm \text { SD } \\
\text { Min. }- \text { max } \\
\text { (Range) }\end{array}$ & $\begin{array}{l}53.9 \pm \\
9.6 \\
(36-66)\end{array}$ & $\begin{array}{l}55 \pm \\
13.9 \\
(29-73)\end{array}$ & 0.85 \\
\hline \multirow[t]{2}{*}{ Sex } & $\begin{array}{l}\text { Male n } \\
(\%)\end{array}$ & $3(30 \%)$ & $6(75 \%)$ & 0.15 \\
\hline & $\begin{array}{l}\text { Female n } \\
(\%)\end{array}$ & $7(70 \%)$ & $2(25 \%)$ & \\
\hline $\begin{array}{l}\text { Site of } \\
\text { Catheter } \\
\text { insertion n } \\
(\%)\end{array}$ & $\begin{array}{l}\text { Femoral } \\
\text { vein }\end{array}$ & $9(90 \%)$ & $8(100 \%)$ & 1.0 \\
\hline $\begin{array}{l}\text { Duration of } \\
\text { catheterization } \\
\text { (days) }\end{array}$ & $\begin{array}{l}\text { Min. -max } \\
\text { (Range) }\end{array}$ & $\begin{array}{l}53.9 \pm \\
15.1 \\
(32-71)\end{array}$ & $\begin{array}{l}46.5 \pm \\
9.6 \\
(36-60)\end{array}$ & 0.25 \\
\hline
\end{tabular}

FET test was used $\quad{ }^{*} P<0.05$ was considered significant.

From table (3), there was no statistically significant difference between CRBSI and Non-CRBSI patients regarding sex, age, site of catheter insertion and duration of catheterization.

\section{Discussion:}

In the present study, the aim was to assess the use of SCs in CRBSIs in asymptomatic HD patients. We found the incidence density of the CRBSI in the present study was 10.8 per 1000 catheter days. This result agrees with the previous study which showed that 
the incidence of CRBSI was 10.8 per 1000 catheter days [6].

In contrast to our result, several studies reported that the incidence of CRBSI was 0.27 episodes per 1,000 catheter days [1]. Moreover, recent data from Canada shows a CRBSI incidence of less than 0.5 per 1000 catheter days [7].

Other studies have reported CRBSI rates ranging from 0.41 to 1.7 per 1000 catheter days and this also is not in agreement with our results [8]. Other studies, reported, the incidence of CRBSI ranges from 2.3 to 16.8 episodes per 1000 catheter-days [9]. So, the reported incidence of CRBSI is variable from country to country and even hospital to hospitals [10].

In studies concerned with CRBSI in Egypt in different centers, the incidence of CRBSI was variable; the incidence of CRBSI in the study conducted in the National Heart Institute was 7.95 per 1000 catheter days [11]. A conducted study on ICUs of Zagazig University Hospitals showing the incidence of CRBSI was 6.01 per 1000 catheter days [12].

These differences in the incidence of CRBSI in different studies may be explained by difference in the type of catheter, site of catheter insertion, number of lumens, frequency of catheter manipulation, lack of compliance to infection control guidelines shortage of resources in developing countries and patient-related factors, such as underlying disease and severity of illness as patients in our study were hemodialysis patients who are immunocompromised and kept the CVCs for long period with increased the chance for the incidence of CRBSI.

In the present study, a total of $60 \mathrm{SCs}$ were collected with a mean number per patient of 3.3. Of these $24(40 \%)$ were negative (group 1), and $36(60 \%)$ gave positive results [0 $(0 \%)$ in group 2, $6(10 \%)$ in group 3, and 30 $(50 \%)$ in group 4]. In contrast to our results, another study carried out, a total of 1.734 SCs were collected with the median number per patient 18. Of which $94.2 \%$ were negative and $5.8 \%$ were positive $(4.6 \%$ group $2,0.7 \%$ group 3 and $0.5 \%$ as group 4 ) [1].

In the present study, CRBSI associated microorganisms were $80 \%$ Gram-positive and 20\% Gram-negative. The commonest pathogens causing CRBSI were CoNS $40 \%$, S. aureus $40 \%$, E coli $10 \%$ and Acinetobacter $10 \%$. These results agree with previous studies that showed that $50 \%$ to 
$75 \%$ of the causative organisms of bacteremia in HD patients were Grampositive bacteria, and the remaining less than $25 \%$ were Gram-negative, and S. aureus was the most common causative organism [2]. Also, the previous study was showed that the organisms responsible for dialysis-related CRBSI were Gram-positive in two-thirds of cases, predominantly S. epidermidis, and S. aureus [13]. However, several studies differed from our results and reported that the Gram-negative organisms were predominant over Gram-positive bacteria $[14,15]$.

On analysis of risk factors for CRBSI in the current study, it was found that no significant association between the age of the patients and the occurrence of CRBSI. These results agree with previous studies that found no significant association between the age of the patients and the occurrence of CRBSI $[16,15]$. In contrast, several studies showed a significant association of CRBSI with increasing age $[17,18]$.

As regards the sex and its association with CRBSI, our study showed no significant association between sex and CRBSI, we found one study agreed with this result [15]. In contrast, another study showed an association between CRBSI and female sex but not with male sex [16]. Male sex was reported to have a significant association of CRBSI, and this is not in agreement with our results [18]. As regards the duration of catheterization, our study showed that no significant association between the duration of catheterization and the occurrence of CRBSI. These results agree with previous studies $[\mathbf{1 9 , 2 0}$. In contrast to our results, several studies declared a highly significant association between the duration of catheterization and CRBSI $[\mathbf{2 1}, \mathbf{2 2}, \mathbf{6}]$.

Also, another study reported that it has been proposed that the risk of CRBSI increases with increasing duration of catheterization [23]. Also, a different study mentioned that fewer CVCs would predispose to reduced infection incidence, but longer catheter placement durations would predispose to increased incidence [24].

The question of which venous catheterization site is associated with the higher risk of infection remains controversial. In the current study, the incidence of CRBSI was $100 \%$ (1/1) when the catheter was inserted in the femoral vein access, but it was $52.9 \%$ (9/17) when the catheter was inserted in internal jugular vein access, but the net result of this study, there 
is no significant association between the site of catheter insertion and the occurrence of CRBSI.

In agreement with our result, several studies detected no significant association between the site of catheter insertion and the occurrence of CRBSI $[16,6,19,20]$. A meta-analysis showed that the average CRBSI density was 2.5 per 1,000 catheter days regardless of location showing no difference in the rate of CRBSIs between the three sites of catheter insertion, which is also in agreement with our results [25].

In contrast, the CVC insertion site was an independent risk factor for CRBSI in a previous study [26]. Also, a different study showed that the incidence of CRBSI associated with non-tunneled catheters was highest for femoral catheters, followed by internal jugular catheters then subclavian catheters [27].

A previous study showed that femoral venous access had a significant rate of CRBSI than the peripheral and subclavian access this is probably due to a greater degree of bacterial colonization of the groin compared to the shoulder and neck [28].

\section{Conclusion,}

Our findings suggest that SCs based on easily accessible samples can be used to prevent CRBSI. Nevertheless, the most important strategy to avoid catheter-related infection is adherence to the written protocol and training of staff in HD units. Our triage protocol could be useful for individual HD patients at high risk of infection who can benefit from ALT interventions.

\section{References:}

1. Brañas P, Morales E, Rios F, Sanz F, Gutiérrez E, Quintanilla N, et al. Usefulness of endoluminal catheter colonization surveillance cultures to reduce catheter-related bloodstream infections in hemodialysis. Am J Infect Control. 2014;42(11):1182-7.

2. Suzuki M, Satoh N, Nakamura M, Horita S, Seki G, Moriya K. Bacteremia in hemodialysis patients. World J Nephrol. 2016;5(6):489- 96.

3. Chaves F, Garnacho-Montero J, Del Pozo JL, Bouza E, Capdevila JA, De Cueto M, et al. Diagnosis and treatment of catheter-related bloodstream infection: Clinical guidelines of the Spanish Society of Infectious Diseases and Clinical Microbiology and (SEIMC) and the Spanish Society of Intensive and Critical Care Medicine A. Med intensive. 2018;42(1):5-36.

4. Böhlke M, Uliano G, Barcellos FC. Hemodialysis catheter-related infection: prophylaxis, diagnosis, and treatment. J Vasc Access. 2015;16(5):347-55.

5. Garcia X, Sabatier C, Ferrer R, Fontanals D, Duarte M, Colomina M, et al. Differential time to 
positivity of blood cultures: A valid method for diagnosing catheter-related bloodstream infections in the intensive care unit. Med Intensiva (English Ed. 2012;36(3):169-76.

6. Sahli F, Feidjel R, Laalaoui R. Hemodialysis catheter-related infection: rates, risk factors, and pathogens. J Infect Public Health. 2017;10(4):403-8.

7. Dumaine CS, Brown RS, MacRae JM, Oliver MJ, Ravani P, Quinn RR. Central venous catheters for chronic hemodialysis: Is last choice never the right choice? In: Seminars in dialysis. 2018; 31:310.

8. Vashi PG, Virginkar N, Popiel B, Edwin P, Gupta D. Incidence of and factors associated with catheter-related bloodstream infection in patients with advanced solid tumors on home parenteral nutrition managed using a standardized catheter care protocol. BMC Infect Dis. 2017;17(1):37280.

9. Eggimann P, Pittet D. Overview of catheterrelated infections with special emphasis on prevention based on educational programs. Clin Microbiol Infect. 2002;8(5):295-309.

10. Gahlot R, Nigam C, Kumar V, Yadav G, Anupurba S. Catheter-related bloodstream infections. Int J Crit Illn Inj Sci. 2014;4(2):162-7.

11. Azqul M., Foda M., Mahmoud Gh, and Gaddoue I. Incidence, risk factors and microbial profile of central catheter related blood stream infection: A six months prospective study at cardiac care units, National Heart Institute. Thesis for a master's degree in public health and Community Medicine, Faculty of Medicine, Zagazig University (2018).

12. Abdelsalam N, El Nemr W, Fahmy H, and Abed El Razek GH. An interventional Study to
Decrease Central Venous Catheter Related Blood Stream Infection in Intensive Care Units, Zagazig University Hospital, MD thesis in Public Health and Community Medicine, Faculty of Medicine, Zagazig University (2013).

13. Katneni R, Hedayati SS. Central venous catheter-related bacteremia in chronic hemodialysis patients: epidemiology and evidence-based management. Nat Clin Pract Nephrol. 2007;3(5):256-66.

14. Sundaresan S, Vincent S, Sivasubramanian S, Jayaraj SS. A study on bacteriological profile of blood culture sample from dialysis patients. South Indian J Biol Sci. 2015;1(3):151-5.

15.Wang T-Y, Lee K-D, Chen P-T, Chen M-C, Chen Y-Y, Huang C-E, et al. Incidence and risk factors for central venous access port-related infection in Chinese cancer patients. J Formos Med Assoc. 2015;114(11):1055-60.

16.Inamdar DP, Taklikar S, Baveja S. Universal Precautions in Preventing Central Venous Catheter Associated Blood Stream Infections. A Study at Tertiary Care Hospital of Central Line Bundles in MICU. Int J Curr Microbiol App Sci. 2018;7(1):2484-91.

17.Powe NR, Jaar B, Furth SL, Hermann J, Briggs W. Septicemia in dialysis patients: incidence, risk factors, and prognosis. Kidney Int. 1999;55(3):1081-90.

18. Saxena AK, Panbotra BR. Haemodialysis catheter-related bloodstream infections: current treatment options and strategies for prevention. Swiss Med Wkly. 2005;135(9-10):127-38.

19. Kumar A, Sharma RM, Jaideep CN, Hazra N. Diagnosis of central venous catheter-related 

Blood stream infection without catheter removal: A prospective observational study. Med J Armed Forces India. 2014;70(1):17-21.

20. Gowardman JR, Montgomery C, Thirlwell S, Shewan J, Idema A, Larsen PD, et al. Central venous catheter-related bloodstream infections: an analysis of incidence and risk factors in a cohort of 400 patients. Intensive Care Med. 1998;24(10):1034-9.

21. Dimick JB, Swoboda S, Talamini MA, Pelz RK, Hendrix CW, Lipsett PA. Risk of colonization of central venous catheters: catheters for total parenteral nutrition vs other catheters. Am J Crit Care. 2003;12(4):328-35.

22. Parameswaran R, Sherchan JB, Varma M, Mukhopadhyay C, Vidyasagar S. Intravascular catheter-related infections in an Indian tertiary care hospital. J Infect Dev Ctries. 2010;5(6):452-8.

23. Curtis R. Catheter-related bloodstream infection in the intensive care unit. J Intensive Care Soc. 2009;10(2):102-8.

24.Longmate AG, Ellis KS, Boyle L, Maher S, Cairns CJS, Lloyd SM, et al. Elimination of central-venous-catheter-related bloodstream infections from the intensive care unit. BMJ Qual Saf. 2011;20(2):174-80.

25. Marik PE, Flemmer M, Harrison W. The risk of catheter-related bloodstream infection with femoral venous catheters as compared to subclavian and internal jugular venous catheters: a systematic review of the literature and metaanalysis. Crit Care Med. 2012;40(8):2479-85.

26. Lemaire X, Morena M, Leray-Moragués H, Henriet-Viprey D, Chenine L, Defez-Fougeron $\mathrm{C}$, et al. Analysis of risk factors for catheterrelated bacteremia in 2000 permanent dual catheters for hemodialysis. Blood Purif. 2009;28(1):21-8.

27. Weijmer MC, Vervloet MG, ter Wee PM. Compared to tunnelled cuffed haemodialysis catheters, temporary untunnelled catheters are associated with more complications already within 2 weeks of use. Nephrol Dial Transplant. 2004;19(3):670-7.

28. Lorente L, Villegas J, Martin MM, Jiménez A, Mora ML. Catheter-related infection in critically ill patients. Intensive Care Med. 2004;30(8):1681-4.

To cite this article: Soheir Abd El- Rahman, Afaf Fathi Khamiss, Saddam H. Abd El- Aziz , Hala A. Abd El- Karim. Role of Endoluminal Catheter Colonization Surveillance Cultures to Reduce Catheter-Related Blood Stream Infections in Hemodialysis Patients. BMFJ 2020; 37(3):542552, DOI: $10.21608 / \mathrm{bmfj} .2020 .21923 .1199$ 